\title{
INFLUÊNCIA DA ALTURA DE COMEDOUROS TUBULARES NO COMPORTAMENTOINGESTIVO DE FRANGOS DE CORTE
}

\author{
EFFECT OF TUBULAR FEEDER HEIGHT ON INGESTIVE BEHAVIOR OF BROILER
}

\author{
Roll, V.F.B. ${ }^{1 \star}$, Dai Prá, M.A. ${ }^{2}$, Roll, A.A.P. ${ }^{1}$, Xavier, E.G. ${ }^{1}$, Rossi, P. ${ }^{1}$, Anciuti, M.A. ${ }^{3}$ e Rutz, F. ${ }^{1}$ \\ ${ }^{1}$ Departamento de Zootecnia. Faculdade de Agronomia Eliseu Maciel. FAEM-UFPel. Campus Universitário \\ s/n. Cx. Postal 354. CEP 96010-900. Pelotas, RS. Brasil. *roll98@ufpel.edu.br \\ ${ }^{2}$ Brasil Foods S/A. Av. Presidente Vargas, 1040. CEP 99150-000. Marau, RS. Brasil. marcos.pra@ \\ brasilfoods.com \\ ${ }^{3}$ Conjunto Agrotécnico Visconde da Graça. CAVG-UFPEL. Av. Idelfonso Simões Lopes, 2791. Cx. Postal \\ 460. CEP 96060-290. Brasil.
}

\section{PaLAVRAS ChaVE ADICIONAIS}

Motivação. Desempenho. Teste de preferência.

\section{RESUMO}

O objetivo deste trabalho foi de verificar o efeito da altura de comedouros tubulares sobre o comportamento de frangos de corte. Foram alojados 880 frangos de corte fêmeas Ross divididos em 40 boxes (repetições) com 22 aves cada (11 aves $/ \mathrm{m}^{2}$ ). Nos boxes, a cama foi de cepilho de madeira, com bebedouros lineares de água corrente e comedouros tubulares com capacidade para $18 \mathrm{~kg}$ de ração. As aves foram submetidas a duas alturas de comedouro em dois períodos diferentes (28-35 dias de idade, comedouro alto (CAL): borda superior do comedouro com $20 \mathrm{~cm}$ do solo, comedouro baixo (CB): borda superior com $11 \mathrm{~cm}$ do solo; 36 a 42 dias de idade - CAL: borda superior com $25 \mathrm{~cm}$ do solo, CB: borda superior com $18 \mathrm{~cm}$ do solo). Para verificar a descrição geral do comportamento das aves foi utilizada a técnica de amostragem instantânea scan sampling e no estudo do comportamento de ingestão das aves (monitorado durante $12 \mathrm{~h}$ consecutivas) utilizou-se como ferramenta de registro microcâmeras instaladas simultaneamente em 4 boxes. No teste de preferência os frangos individualmente ou coletivamente deveriam escolher entre um comedouro alto ou um comedouro baixo disponíveis ao mesmo tempo dentro do box. Foi utilizado um delineamento completamente casualizado sendo que as médias das variáveis respostas foram comparadas através do teste $t$ ou estatística não paramétrica quando necessária.

Recibido: 11-1-08. Aceptado: 19-6-08.

\author{
AdDitional KEYWORDS \\ Motivation. Performance. Preference test.
}

Os frangos de corte preferem consumir ração em comedouros tubulares regulados a baixa altura. A utilização de comedouros baixos reduz a freqüência de visitas ao mesmo, sendo inversamente proporcional ao tempo de ingestão de alimentos, porém sem afetar ao consumo total de ração.

\section{SUMMARY}

This paper aimed to study the effect of tubular feeder height on ingestive behavior of broiler. Eight hundred and eighty female broilers (Ross) were randomly allotted to 40 pens (replications) with 22 broilers each $\left(11\right.$ birds $\left./ \mathrm{m}^{2}\right)$. Each experimental pen contained wood shavings, linear waterers and tubular feeders (18 kg capacity). Birds were subjected to two feeders height in two periods (28 to 35 days of age, feeder height $(\mathrm{FH})$ : superior edge $20 \mathrm{~cm}$ height; feeder low $(\mathrm{FL})$ : superior edge $11 \mathrm{~cm}$ height; 36 to 42 days of age, $\mathrm{FH}$ : superior edge $25 \mathrm{~cm}$ height; FL: superior edge $18 \mathrm{~cm}$ height). Scan sampling technique was used to study the general description of avian behavior, while micro cameras were simultaneously installed in 4 pens to study ingestive behavior (12 hours non-stop monitoring). In the preference test the broilers should individually or collectively choose between either a high or a low feeder, with both available at the same time inside the pen. A completely randomized design was used and $t$ test

Arch. Zootec. 59 (225): 115-122. 2010. 
was utilized to compare the treatment averages. Non-parametric statistic was employed when necessary. Results showed that broilers prefer to consume feed from low feeders. The use of low height feeders reduces the frequency of visits for eating and is inversely correlated to the time needed for eating, but it does not affect feed consumption.

\section{INTRODUÇÃO}

Praticamente não há registro, na literatura científica, de trabalhos que relacionem altura de comedouro e comportamento ingestivo em frangos de corte. Apesar disso, a grande maioria das guias de manejo de frangos de corte recomenda ajustar o comedouro à altura do dorso das aves visando, principalmente, reduzir o desperdício de ração.

Segundo Ferket e Gernat (2006) os comedouros devem ser ajustados a uma altura em que todas as aves possam ter fácil acesso ao alimento. Comedouros que são regulados muito altos podem dificultar o consumo de ração pelas aves menores e aumentar a desuniformidade do lote.

O conhecimento do comportamento dos animais traz consigo uma série de vantagens, entre as quais se inclui a possibilidade de melhorar aspectos de manejo (Roll et al., 2006). É sabido que as condições de manejo podem afetar os níveis de estresse (Gibson et al., 1986). Murphy ePreston(1988) verificaram que aves recebendo menos alimento nos comedouros tornam-se mais competitivas e alteram seu comportamento ingestivo.
Porém, sabe-se que o comportamento é um fenômeno complexo, pois ocorre tanto em indivíduos isolados como em grupos, sendo controlado através de mecanismos neurobiológicos e hormonais (Mench, 1992). Um dos métodos de estudo consiste na observação do repertório completo de comportamentos (Webster e Hurnick, 1990). Outros métodos costumam avaliar a normalidade dos comportamentos, as interações sociais, a motivação e as preferências.

O presente trabalho teve por objetivo estudar a influência da regulagem da altura dos comedouros tubulares no comportamento de frangos e a sua interação com aspectos produtivos.

\section{MATERIAL E MÉTODOS}

Foram alojados em aviário experimental 880 frangos de corte fêmeas Ross divididos em 40 boxes (repetições) com 22 aves cada $\left(11\right.$ aves $\left./ \mathrm{m}^{2}\right)$. Nos boxes, a cama foi de cepilho de madeira, com bebedouros lineares de água corrente e comedouros tubulares com capacidade para $18 \mathrm{~kg}$ de ração.

No período de 28 a 42 dias de idade as aves recebiam ração de crescimento com 18,0\% de proteína bruta e $2950 \mathrm{kcal} \mathrm{EM/kg}$ ração. As aves foram submetidas a duas alturas de comedouro a partir dos 28 dias de idade, conforme esquema apresentado na tabela I.

Para a coleta de dados do comportamento de ingestão das aves foram utilizadas como ferramenta de registro microcâmeras insta-

Tabela I. Esquema da regulagem da altura dos comedouros utilizados no experimento. (Feeder height scheme used in the experiment).

\begin{tabular}{|c|c|c|c|c|c|c|c|c|}
\hline \multirow{4}{*}{$\begin{array}{l}\text { Comedouro } \\
\text { Borda } \\
\text { Altura }(\mathrm{cm})\end{array}$} & \multicolumn{4}{|c|}{ 28-35 dias de idade } & \multicolumn{4}{|c|}{ 36-42 dias de idade } \\
\hline & \multicolumn{2}{|c|}{ Alto } & \multicolumn{2}{|c|}{ Baixo } & \multicolumn{2}{|c|}{ Alto } & \multicolumn{2}{|c|}{ Baixo } \\
\hline & superior & inferior & superior & inferior & superior & inferior & superior & inferior \\
\hline & 20 & 13 & 11 & 4 & 25 & 18 & 11 & 4 \\
\hline
\end{tabular}

Obs: Regulagem interna do comedouro: $4 \mathrm{~cm}$.

Archivos de zootecnia vol. 59, núm. 225, p. 116. 


\section{ALTURA DE COMEDOUROS E COMPORTAMENTO INGESTIVO DE FRANGOS}

ladas simultaneamente em 4 boxes. Neste caso, 4 aves, em cada box, foram identificadas com tinta colorida, e o seu comportamento de ingestão monitorado durante $12 \mathrm{~h}$ consecutivas.

Considerou-se como uma visita o intervalo de tempo desde a chegada da ave ao comedouro até que a mesma retirasse a cabeça de dentro do prato por um período superior a 10 segundos. As imagens registradas foram capturadas através de um aparelho de vídeocassete e utilizadas para determinar a freqüência de visitas e o tempo de permanência das aves nos comedouros.

No primeiro teste de preferência os frangos coletivamente após 2 horas de jejum deveriam escolher entre um comedouro alto ou um comedouro baixo disponíveis ao mesmo tempo dentro do boxe. Os dados foram obtidos através de observação direta do número de aves utilizando os comedouros em intervalos regulares de 2 minutos, durante 1 hora de observação.

No segundo teste de preferência foi colocado e observado no boxe somente um frango de cada vez (total 20 frangos submetidos a 2 horas de jejum). Neste momento eles deveriam escolher entre um comedouro alto ou um comedouro baixo disponíveis ao mesmo tempo dentro do boxe. Através de observação direta foi determinada qual a primeira opção de cada frango e por quanto tempo ele permanecia ingerindo ração, durante um período máximo de 5 minutos de observação.

O cálculo do índice de eficiência produtiva foi realizado em cada tratamento pela fórmula expressa por:

$$
\mathrm{IEP}=\mathrm{Gmd} \times \mathrm{Vb} \times \mathrm{EA}
$$

Emque:

Gmd= peso vivo $(\mathrm{kg}) /$ idade (dias);

$\mathrm{Vb}=$ viabilidade $(\%)=100-(\%)$ mortalidade;

$\mathrm{EA}=$ eficiência alimentar: ganho de peso $(\mathrm{kg}) /$ consumo de ração $(\mathrm{kg})$.

Os dados foram analisados utilizandose um delineamento completamente casuali- zado sendo que as médias das variáveis respostas foram comparadas através do teste $t$ exceto nos testes de preferência onde foi utilizado o teste binomial.

\section{RESULTADOSEDISCUSSÃO}

Ao analisar o efeito da altura de comedouro sobre o comportamento de ingestão dos frangos resultou bastante claro que as aves permaneceram em média o dobro do tempo consumindo ração nos comedouros baixos em comparação com os altos 228 vs. 111,5 segundos, ao mesmo tempo em que a freqüência de visitas aos comedouros baixos foi menor que nos altos (253 vs. 358 p<0,001). Porém, reportando-se aos dados produtivos, é importante salientar que o consumo de ração foi semelhante, indicando a existência de uma correlação negativa entre a freqüência de visitas e a quantidade de alimento ingerido, ou seja, quanto mais vezes o frango vai ao comedouro menos alimento ele consome em cada visita. Aliado a isso, ao se multiplicar a freqüência de visitas pelo tempo de permanência das aves nos comedouros, os resultados esperados seriam 39917segundos [(358visitas x111,5 s) $/ 8$ aves $/ 60 \mathrm{~s}$ ) $=83,13 \mathrm{~min} /$ ave ] e $57684 \mathrm{se}-$ gundos [(253visitas $x 228 \mathrm{~s}) / 8$ aves/60 s)= 120,15 min/ave], em 12 horas de observação, para o tempo total de ingestão nos comedouros altos e baixos, respectivamente.

No entanto, isso não se refletiu em maior consumo de ração propriamente dito, porque as aves nos comedouros baixos, embora permanecessem mais tempo junto aos comedouros, diminuíram a intensidade de consumo, isto é, reduziram o tamanho do bocado por estarem mais tranqüilas e numa posição mais confortável.

Segundo Estevez (1999) os frangos são capazes de adaptar a sua taxa de consumo de acordo as restrições do ambiente. Por exemplo, quando a relação aves por comedouro é muito alta os frangos aumentam o número de partículas ingeridas em cada visita. Por esta razão o tempo de ingestão 
nos comedouros não é um bom estimador da quantidade de alimento ingerido. No entanto, os comedouros regulados próximos ao piso permitem que as aves comam com facilidade, como se estivessem em seu habitat natural, isto é, ciscando no solo, o que é interessante em termos de bem-estar Lima et al. (1996).

Interpretando os dados de outra forma observa-se um número médio de visitas de 44,8 (358visitas/8aves) e de 31,6 (253visitas/8aves) no período de 12 horas de observação, isto é, 3,7 e 2,6 visitas ave/hora nos comedouros altos e baixos, respectivamente.

Esta elevada freqüência de visitas, comparada com outros trabalhos, pode ser em função do desenho do boxe que tinha a forma retangular com o comedouro pendular localizado no centro. Basicamente, em toda a movimentação das aves elas acabavam chegando muito nróximo do comedouro, desta forma estimulando o comportamento ingestivo.

Expressando os dados anteriores em forma de percentagem obtém-se que as aves gastaram $11,5(83,13 / 720 \mathrm{~min})$ e $16,6 \%$ $(120,15 / 720 \mathrm{~min})$ do seu tempo realizando o comportamento ingestivo nos comedouros altos e baixos, respectivamente.

A freqüência de visitas e o tempo de ingestão neste trabalho são maiores que os $11,1 \%$ observados por Cornetto e Estevez (2001) e que os $10 \%$ de Shields et al. (2005) em frangos de corte de diferentes idades.

Segundo o relatório do Scientific Committee on Animal Health and Animal Welfare (2000), quando se aumenta a densidade de aves ocorre que elas passam mais tempo próximas aos comedouros e bebedouros para obter suficiente água e comida. Esta é outra possível explicação para os resultados mais elevados de idas ao

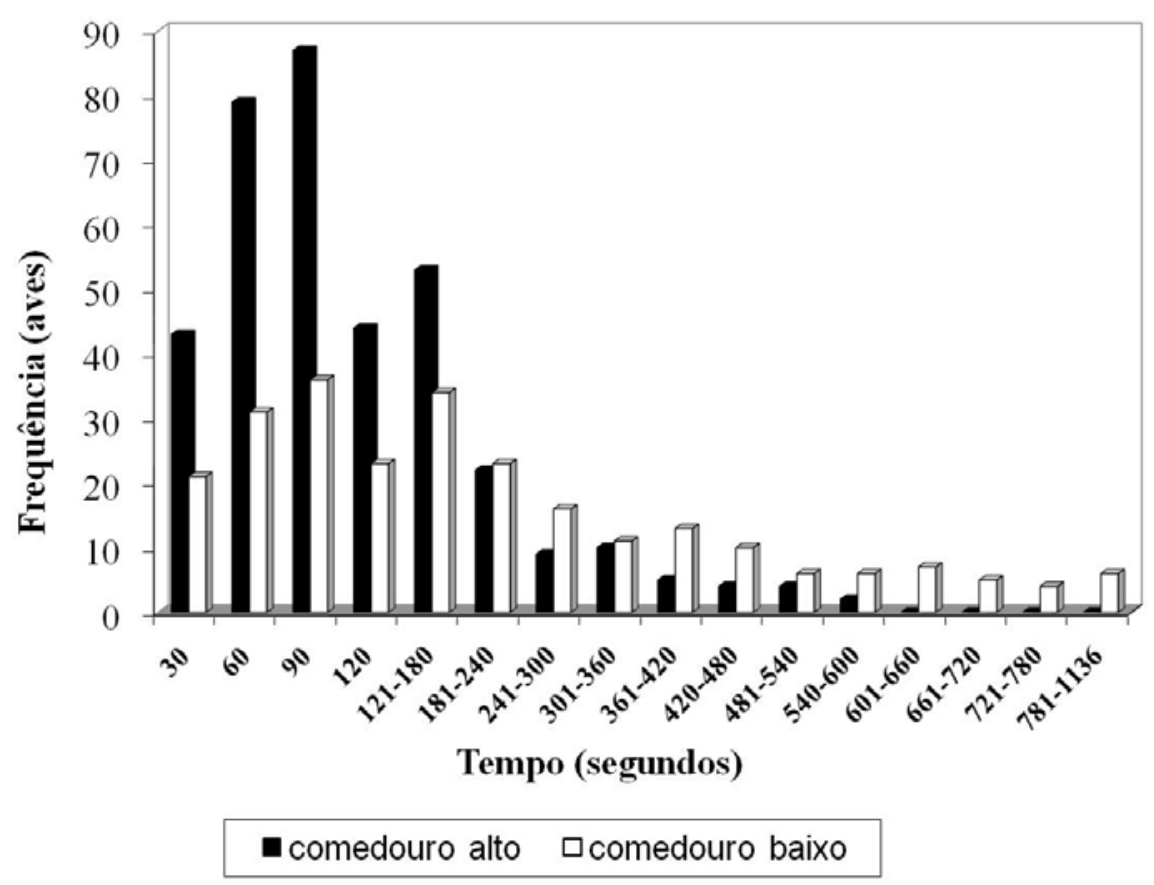

Figura 1. Distribuição do tempo de ingestão de acordo com a altura de comedouro. (Distribution of time of ingestion according to feeder height).

Archivos de zootecnia vol. 59, núm. 225, p. 118. 


\section{ALTURA DE COMEDOUROS E COMPORTAMENTO INGESTIVO DE FRANGOS}

comedouro encontrados neste trabalho, pois a densidade média foi muito próxima a $25 \mathrm{~kg} / \mathrm{m}^{2}$.

No entanto, Bizeray et al. (2002) utilizando a técnica de amostragem scan observaram uma variação de 7,7 a 15,5\% no tempo gasto com a ingestão de alimentos em aves com idades variando entre 9 a 37 dias.

Portanto, parece existir uma variação natural no tempo gasto com o comportamento ingestivo que pode ser influenciado, entre outras coisas, pela forma física do alimento, temperatura ambiente, densidade e idade das aves. As diferentes técnicas de amostragem empregadas na coleta de dados também podem influenciar os resultados o que justificaria, em parte, as diferenças encontradas neste trabalho em relação com a literatura.

Todos esses fatores fazem com que a distribuição percentual dos comportamentos seja equivalente, mas que se expressem de formas diferentes. Por exemplo, nos comedouros baixos um menor número de aves alimenta-se simultaneamente durante mais tempo do que nos comedouros altos, mas ao final o consumo pode ser considerado semelhante nos dois casos. Porém, Ferket e Gernat (2006) afirmam que os comedouros nunca deveriam estar ocupados o dia inteiro porque as aves submissas não consumirão as suas exigências nutricionais.

Verificou-se que a maior parte das aves fez visitas rápidas aos comedouros altos, permanecendo menos do que 180 segundos consumindo ração em cada um deles diferindo significativamente $(\mathrm{p}<0,001)$ das médias encontradas nos comedouros baixos (figura 1). Este resultado concorda com a sugestão de Estevez (1999) de que os frangos permanecem em torno de 3 minutos em cada visita ao comedouro. Observou-se, também, que nenhuma ave permaneceu mais do que 600 segundos (10 min) ininterruptos consumindo ração nos comedouros altos. Já no caso dos comedouros baixos alguns frangos permaneceram até 19 minutos junto ao comedouro.
Yo et al. (1997) verificaram que a duração média de cada acesso ao comedouro depende da forma física da ração variando de 56 segundos quando a ração é peletizada para 114 segundos quando farelada. Estes resultados se aproximam aos encontrados para comedouro alto cuja maior freqüência de visitas foi entre 60 a 120 segundos.

Depois de submeter às aves a um período de jejum de 2 horas, com o objetivo de forçar a sua ida aos comedouros, verificouse que os frangos preferiram consumir ração em comedouros tubulares regulados numa posição mais baixa do que a recomendada na maioria das guias de manejo, isto é, na altura do dorso das aves.

Observou-se que o número de aves que preferiram consumir ração no comedouro baixo foi sempre superior em comparação ao alto $(\mathrm{p}<0,001)$.

$\mathrm{O}$ animal se encontra em situação de bem-estar quando está em equilíbrio consigo mesmo e com o ambiente onde se encontra imerso (Broom, 1986). Assim sendo, o estresse ou uma restrição de uma preferência pode ter efeitos negativos sobre a eficiência produtiva (produtividade) e o bem-estar animal.

No início observou-se que certo número de aves optou por utilizar o comedouro alto, pois a prioridade máxima naquele momento era saciar a fome, mas com o passar do tempo, já saciada a fome, as aves tornaramse mais seletivas e começaram a mostrar suas preferências secundárias (figura 2). Neste caso, observou-se que após 20 minutos todos os frangos estavam consumindo ração no comedouro baixo e nenhum no alto $(\mathrm{p}<0,0001)$. Segundo Dawkins (1988), os animais recusam o que lhes provoca dor ou mal-estar e escolhem o que lhes provoca prazer ou bem-estar.

Observou-se, também, que nos comedouros baixos foi sempre menor o número de aves comendo simultaneamente $(\mathrm{p}<0,0001)$, e isto ocorreu porque as aves permaneceram numa posição que ocupa mais espaço, ao mesmo tempo em que as deixava mais 


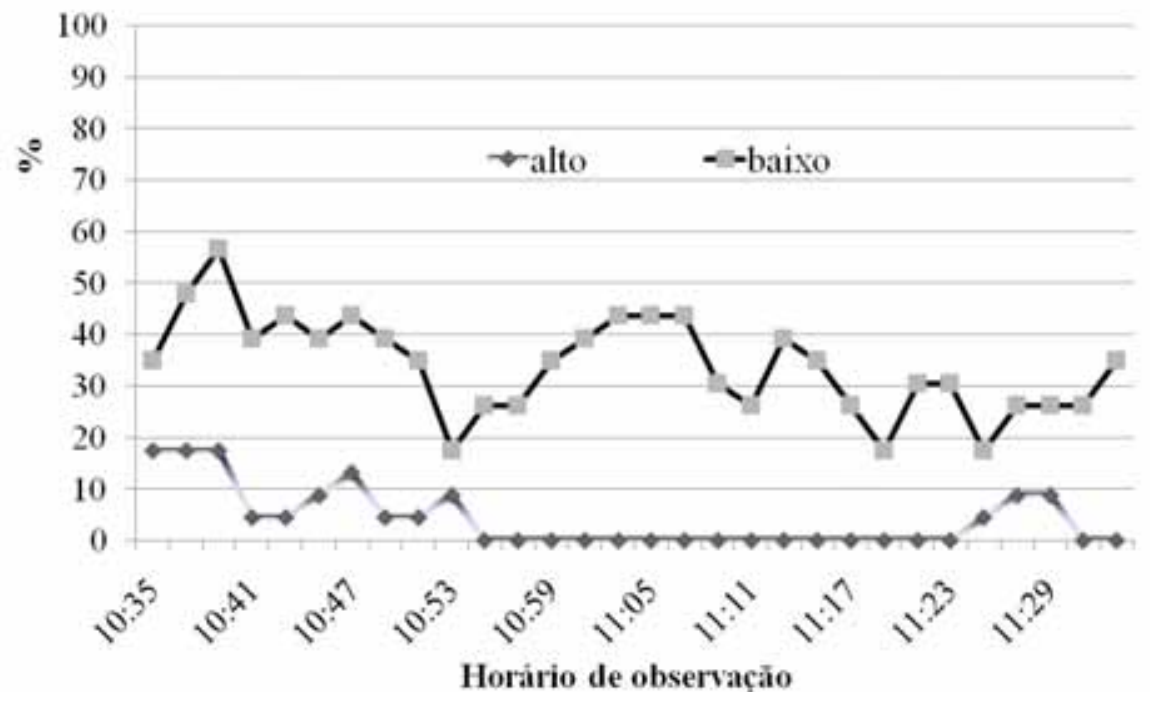

Figura 2. Porcentagem de aves utilizando os comedouros presentes simultaneamente em quatro boxes em duas alturas, após 2 horas de jejum. (Percentage of birds using either a high or a low feeder simultaneously installed in four pens, after two hours of feed restriction).

estáveis, diminuindo a possibilidade de serem empurradas para fora do comedouro por suas companheiras, a exemplo do que ocorreu quando elas estavam em pé.

No teste de preferência em que dois comedouros estavam presentes dentro do box, $85 \%$ das aves, individualmente, tiveram como primeira opção consumir ração no comedouro baixo e apenas $15 \%$ optaram por consumir ração no comedouro alto, com $\mathrm{p}<0,0001$, pelo teste binomial. Das aves que inicialmente optaram pelo comedouro baixo, apenas $23 \%$ mudaram para comedouro alto. No entanto, das aves que no primeiro momento optaram por comedouro alto, $100 \%$ passaram a comer no comedouro baixo. Estes resultados indicam a forte preferência que as aves têm por comedouros regulados à baixa altura comparado com os regulados na altura do seu dorso.

Uma preferência se caracteriza pela escolha por parte do animal da qualidade e quantidade de um recurso determinado, sendo que neste caso a demanda elástica, ou seja, a privação desse recurso não impli- ca no aparecimento de comportamentos anormais. Por outro lado, uma necessidade corresponde a um tipo de recurso preferido pelo animal, cuja demanda é inelástica e a falta do recurso provoca o aparecimento de comportamentos anormais (De Jong, 2007). Como exemplo de uma necessidade pode-se citar o alimento pelo qual o animal normalmente se dispõe a trabalhar ou a fazer um esforço maior para satisfazer a sua motivação pela ingestão do mesmo. Os resultados do presente trabalho são úteis para determinar a preferência das aves, semelhante ao trabalho de Sanotra et al.(1995) que testaram a preferência das aves por diferentes materiais de cama.

Com relação aos dados de desempenho não foram encontradas diferenças significativas entre os tratamentos (tabela II). Tudo indica que as aves ajustam o tamanho do bocado de acordo com o tempo em que permanecem nos comedouros finalizando em consumos de ração e desempenho semelhantes.

No entanto, comedouros ajustados próximos ao piso permitem que todas as

Archivos de zootecnia vol. 59, núm. 225, p. 120. 
Tabela II. Desempenho de acordo com a altura de comedouro no período de 28 a 42 dias de idade (médias \pm erro). (Performance of broilers (28 to 42 days of age) according to feeder height (means \pm error)).

\begin{tabular}{lcccccc}
\hline & \multicolumn{3}{c}{28 a 36 dias } & & & 36 a 42 dias \\
& CAL & CB & Prob* & CAL & CB & Prob* $^{*}$ \\
\hline Clave/dia (g) & $132,8 \pm 1,2$ & $132,4 \pm 0,9$ & NS & $190,9 \pm 1,4$ & $184,4 \pm 1,42$ & NS \\
Caj/ave/dia (g) & $132,7 \pm 1,2$ & $132,2 \pm 0,9$ & NS & $190,6 \pm 1,4$ & $184,2 \pm 1,4$ & NS \\
PV/sem (g) & $1915,5 \pm 8,4$ & $1938,6 \pm 10,0$ & NS & $2381,7 \pm 12,2$ & $2395,6 \pm 13,0$ & NS \\
GP/sem & $590,5 \pm 3,9$ & $613,3 \pm 4,4$ & NS & $466,3 \pm 5,7$ & $457 \pm 5,9$ & NS \\
GM/sem & $84,4 \pm 0,6$ & $87,6 \pm 0,6$ & NS & $66,6 \pm 0,8$ & $65,3 \pm 0,8$ & NS \\
CA/sem & $1,57 \pm 0,01$ & $1,51 \pm 0,01$ & NS & $2,87 \pm 0,02$ & $2,83 \pm 0,03$ & NS \\
IEP/sem & $343,1 \pm 4,3$ & $360,1 \pm 5,2$ & NS & $195,2 \pm 2,8$ & $197,9 \pm 3,9$ & NS \\
\hline
\end{tabular}

CAL: comedouro alto; CB: comedouro baixo; C: consumo; Caj: consumo ajustado para mortalidade; PV: peso vivo; GM: ganho médio; Sem: semana; CA: conversão alimentar; IEP: índice de eficiência produtiva. *Teste $t$.

aves, inclusive as menos desenvolvidas, possam comer com mais facilidade, o que é recomendável do ponto de vista do bemestar animal.

\section{CONCLUSÕES}

Os frangos de corte preferem consumir

\section{BIBLIOGRAFIA}

Bizeray, D., Leterrier, C., Constantin, P., Picard, M. and Faure, J.M. 2002. Sequential feeding can increase activity and improve gait score in meattype chickens. Poultry Sci., 81: 1798-1806.

Broom, D.M. 1986. Indicators of poor welfare. Brit. Vet. J., 142: 524-526.

Cornetto, T. and Estevez, I. 2001. Behavior of the domestic fowl in the presence of vertical panels. Poultry Sci., 80: 1455-1462.

Dawkins, M.S. 1988. Behavioural deprivation: A central problem in animal welfare. Appl. Anim. Behav. Sci., 20: 200-225.

De Jong, I.C., Wolthuis-Fillerup, M. and Van Reenen, C.G. 2007. Strength of preference for dustbathing and foraging substrates in laying hens. Appl. Anim. Behav. Sci., 104: 24-36.

Estevez, I. 1999. Bird density: How it can affect the behavior and health of your flock. In: Production management featured articles. Disponível em: <http://www.thepoultrysite.com/articles/453/ ração em comedouros tubulares regulados a baixa altura.

A utilização de comedouros baixos reduz a freqüência de visitas ao mesmo, sendo inversamente proporcional ao tempo de ingestão de alimentos, porém sem afetar ao consumo total de ração.

bird-density-how-it-can-affect-the-behaviorand-health-of-your-flock>. Acesso: 20 dez. 2007.

Ferket, P.R. and Gernat, A.G. 2006. Factors that affect feed intake of meat birds: A Review. Int. J. Poultry Sci., 5: 905-911

Gibson, S.W., Hughes, B.O., Harvey, S. and Dun, P. 1986. Plasma concentrations of corticosterone and thyroid hormones in laying fowls from different housing systems. Brit. Poultry Sci., 27: 621-628.

Lima, C.A.R., Salles, G.S. e Curvello, F.A. 1996. Efeito do uso do óleo em rações de frangos de corte no verão. UFRJ Prêmio Lamas 1996. In: Conferência APINCO de Ciência e Tecnologia Avícola. Curitiba. Brasil. p. 45.

Mench, J.A. 1992. Applied ethology and poultry production. Poultry Sci., 71: 631-633.

Murphy, L.B. and Preston, A.P. 1988. Food availability and the feeding and drinking behaviour of broiler chickens grown commer-

Archivos de zootecnia vol. 59, núm. 225, p. 121. 


\section{ROLL, DAI PRÁ, ROLL, XAVIER, ROSSI, ANCIUTI E RUTZ}

cially. Brit. Poultry Sci., 29: 273-283.

Roll, V.F.B., Rech, C.L. de S., Xavier, E.G., Rech, J.L., Rutz, F. e Del Pino, F.A.B. 2006. Comportamento animal, conceitos e técnicas de estudo. Editora e Gráfica Universitária. UFPEL. Pelotas. 110 pp.

Sanotra, G.S., Vestergaard, K.S., Agger, J.F. and Lawson, L.G. 1995. The relative preferences for feathers, straw, word-shavings and sand for dustbathing, pecking and scratching in domestic chicks. Appl. Anim. Behav. Sci., 43: 263-277.

Shields, S.J., Garner, J.P. and Mench, J.A. 2005. Effect of sand and wood-shavings bedding on the behavior of broiler chickens. Poultry Sci. 84:
1816-1824

Scientific Committee on Animal Health and Animal Welfare. 2000. The welfare of chickens kept for meat production (broilers). Adopted 21 March 2000. Disponível em http://www.ec.europa.eu/ food/fs/sc/scah/out39_en.pdf. Acesso em 24/ 05/2008.

Webster, A.B. and Hurnick, J.F. 1990. An ethogram of White Leghorn - type hens in battery cages. Can. J. Anim. Sci., 70: 751-760.

Yo, T., Siegel, P.B., Guerin, H. and Picard, M. 1997. Self-selection of dietary protein and energy by broilers grown under a tropical climate: Effect of feed particle size on the feed choice. Poultry Sci., 76: 1467-1473.

Archivos de zootecnia vol. 59, núm. 225, p. 122. 